\title{
Análisis productivo de la suplementación con ensilado de maíz en sistemas ganaderos de doble propósito
}

\section{Productive analysis of the supplementation with corn silage in double-purpose livestock systems}

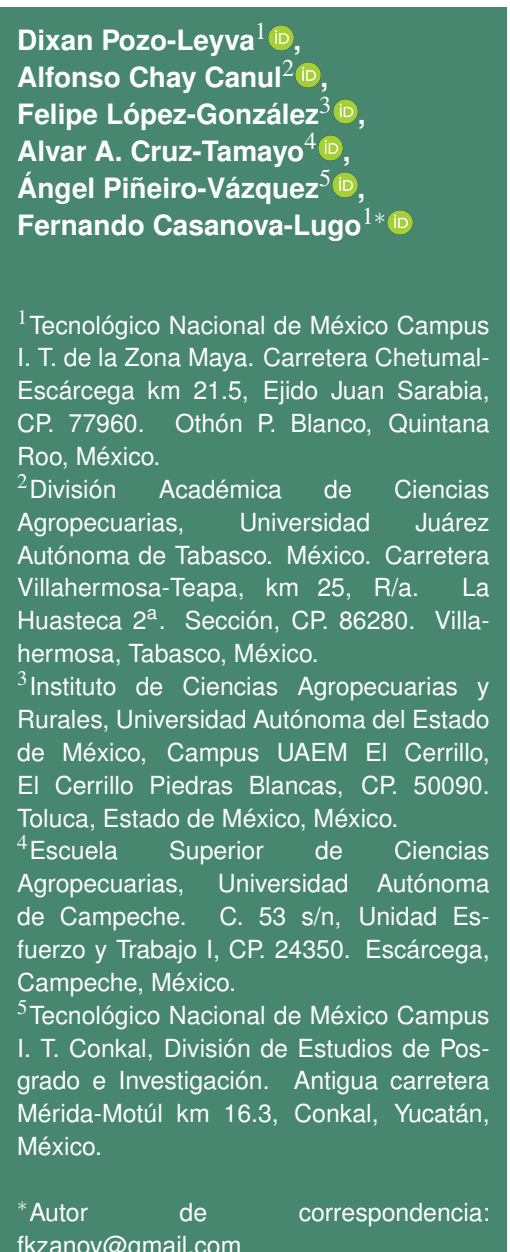

fkzanov@gmail.com

Artículo científico

Recibido: 29 de julio 2021

Aceptado: 22 de noviembre 2021

Como citar: Pozo-Leyva D, Chay Canul A, López-González F, Cruz-Tamayo AA, Piñeiro-Vázquez A, Casanova-Lugo $F$ (2021) Análisis productivo de la suplementación con ensilado de maíz en sistemas ganaderos de doble propósito. Ecosistemas y Recursos Agropecuarios 8(3): e3092. DOI: 10.19136/era.a8n3.3092
RESUMEN. El déficit de forrajes tropicales en la temporada seca requiere de estrategias de alimentación alternas que involucren trasferencia de tecnologías y la conservación de alimentos locales. El objetivo fue evaluar la respuesta animal y el costo beneficio ante raciones mixtas con diferentes niveles de inclusión de ensilado de maíz en sistemas de producción doble propósito del sur de Quintana Roo. Se utilizaron nueve vacas lactantes con 152 días en leche en un diseño experimental de cuadrado latino $3 \times 3$ por triplicado. Las secuencias de tratamiento fueron asignadas al azar con periodos experimentales de 12 días, el tratamiento uno (T1) estuvo conformado por la inclusión del 15\% de ensilado maíz; el T2 por 31\%, T3 por un $45 \%$. Se evaluó la producción de leche, peso vivo, condición corporal, la composición química de la leche y la viabilidad económica. La producción de leche promedio fue de $4.4 \mathrm{~kg} \mathrm{vaca}^{-1} \mathrm{día}^{-1}$, con un contenido de grasa de $3.3 \%$ y proteína cruda de $3.4 \%$. El peso vivo promedio fue de $424.6 \mathrm{~kg}$ y la condición corporal de 2.3, manteniéndose constante a lo largo del experimento. Solo se observaron diferencias $(P<0.05)$ para la condición corporal siendo favorable para T3. La incorporación del ensilado de maíz en un 15, 31 o 45\% vaca ${ }^{-1}$ día $^{-1}$ en las raciones mixtas de vacas doble propósito que producen $4.4 \mathrm{~kg} \mathrm{leche}^{-1}$ día $^{-1}$ no incrementó la producción de leche, pero si tienen un efecto en los costó total de alimentación.

Palabras clave: Alternativas de alimentación, ganadería familiar, raciones mixtas, producción de leche.

ABSTRACT. The deficit of tropical forages in the dry season requires alternative feeding strategies that involve technology transfer and the conservation of local foods. The objective was to evaluate the animal response and the cost of benefit to mixed rations with different levels of inclusion of corn silage in dual-purpose production systems in southern Quintana Roo. Nine lactating cows with 152 days in milk were used in a $3 \times 3$ Latin square experimental design in triplicate. The treatment sequences were randomly assigned with experimental periods of 12 days, treatment one (T1) consisted of the inclusion of $15 \%$ of corn silage; T2 by $31 \%$, T3 by $45 \%$. Milk production, live weight, body condition, chemical composition of milk and economic viability were evaluated. Average milk production was 4.4 $\mathrm{kg} / \mathrm{cow}^{-1} / \mathrm{day}^{-1}$, with a fat content of $3.3 \%$ and crude protein of $3.4 \%$. The average live weight was $424.6 \mathrm{~kg}$ and the body condition of 2.3 , remaining constant throughout the experiment. Differences $(P<0.05)$ were only observed for body condition, being favourable for T3. The incorporation of corn silage in 15, 31 or $45 \%$ $\mathrm{cow}^{-1}$ day $^{-1}$ in the mixed rations of dual-purpose cows that produce $4.4 \mathrm{~kg} \mathrm{milk}^{-1}$ day $^{-1}$ did not increase milk production, but it did they have an effect on total feed costs.

Key words: Food alternatives, family farming, mixed rations, milk production. 


\section{INTRODUCCIÓN}

En la última década, México se encuentra entre los 10 países de mayor producción de carne y leche, ocupando el octavo lugar en función al número de cabezas de ganado. En producción de leche de bovino, ocupa el 16 lugar a nivel mundial, con una producción de 12005692 miles de toneladas, lo que representa el $2 \%$ de la producción total. En México, los sistemas doble propósito son importantes dado que se llevan a cabo en más de 48 millones de hectáreas y concentran el $45 \%$ del inventario nacional de bovinos (SIAP, 2014). De hecho, la ganadería es una de las principales actividades económicas de las zonas rurales de México, donde además de los forrajes disponibles regionalmente, se utilizan subproductos agro-industriales para la alimentación animal como una alternativa para cubrir las necesidades nutricionales (Rojo-Rubio et al. 2009, Albarrán-Portillo et al. 2015). Estos sistemas productivos constituyen una de las principales fuentes de sustento para los productores agropecuarios locales, ya que proporcionan el $20 \%$ de la leche y el $45 \%$ de la carne consumida (Rojo-Rubio et al. 2009), fundamentalmente en los mercados locales, de tal forma permite ingresos diarios a las familias productoras (EspinozaOrtega et al. 2007).

En las zonas tropicales la estacionalidad tiene un efecto directo en la disponibilidad de alimentos de calidad para el ganado, lo que afecta los indicadores productivos y reproductivos, fundamentalmente en aquellos animales que inicia su periodo de lactancia en la temporada seca (Absalón-Medina et al. 2012). En los sistemas ganaderos de doble propósito la alimentación del ganado representa el $65 \%$ de los costos de producción, por lo que el margen de ganancia de los productores es reducido, luego de cubrir los costos operativos de la producción y las necesidades económicas familiares (Albarrán-Portillo et al. 2015). Una de las características de estos sistemas es la estabulación de los animales de forma ocasional para favorecer el crecimiento del pasto durante la estación seca o cuando las condiciones climáticas son adversas (Arjona-Alcocer et al. 2020).

A pesar de estas limitaciones la producción ganadera es una alternativa viable para transformar alimentos fibrosos y subproductos de cosecha en alimentos de alto valor nutricional para consumo humano (López-González et al. 2020). Pero los desafíos de estos sistemas, son mantener los indicadores reproductivos y productivos de forma constante. Principalmente en la temporada de estiaje, cuando los recursos forrajeros internos son limitados y los costos de alimentación se incrementan, por la compra de suplementos como los concentrados comerciales (Ramírez-Rivera et al. 2019, Pozo-Leyva et al. 2019, Pozo-Leyva et al. 2021). Una alternativa viable para reducir costos de alimentación y mantener los indicadores productivos durante la temporada de estiaje, es el empleo del ensilado de maíz (González-Alcántara et al. 2020). Al incluir ensilado de maíz en las estrategias de alimentación se garantiza el mantenimiento del mayor número de animales por área de tierra durante la temporada de estiaje, que es cuando hay marcada escasez de forrajes y alta dependencia de insumos externos (López-González et al. 2020). Además, el ensilado de maíz tiene mayor digestibilidad de sus componentes nutritivos con respecto al uso de rastrojo o maíz molido, lo que permite establecer estrategias de alimentación para la época de escasez de forrajes (Partida-Hernández et al. 2019). Manteniendo un equilibrio entre los factores económicos, el rendimiento, la calidad de la leche y el mantenimiento de la condición corporal (Ramírez-Rivera et al. 2019). Por lo anterior, el objetivo de esta investigación fue evaluar la respuesta animal y el costo beneficio ante raciones mixtas con diferentes niveles de inclusión de ensilado de maíz en sistemas de producción doble propósito del sur de Quintana Roo.

\section{MATERIALES Y MÉTODOS}

El experimento se llevó a cabo al inicio de la temporada seca del 11 de diciembre del 2020 al 16 de enero del 2021 con una duración de cinco semanas. Para el experimento se emplearon nueve vacas de doble propósito provenientes de diferentes cruces de las razas locales cebúinas (Brahman, Gyr y Guzerat), 
las vacas permanecieron estabuladas durante el experimento. La producción de leche promedio al inicio del experimento fue de $4.9 \mathrm{~kg}_{\text {vaca }}{ }^{-1}$ día $^{-1}$ con contenido de grasa del $3.1 \%$, densidad de $31.9 \%$, PC del $3.4 \%$, SNG de $9.5 \%$, PV de $423 \mathrm{~kg}$, condición corporal de 2.0, y promedio de 152 días en producción de leche. El ordeño fue manual y se llevó a cabo una vez al día. Los becerros permanecieron con las vacas por $7 \mathrm{~h}$ al día y posteriormente se encerraron aparte, la edad promedio de los becerros fue de cinco meses aproximadamente.

La unidad de producción participante se localiza en el municipio de Othón P. Blanco, Quintana Roo, México, ubicado entre las coordenadas $18^{\circ} 30^{\prime} \mathrm{LN}$ y $88^{\circ} 29^{\prime} \mathrm{LO}$. El clima es cálido subhúmedo, con precipitación media anual de $1260 \mathrm{~mm}$. Las temperaturas máximas anuales van de 32 a 36 ${ }^{\circ} \mathrm{C}$, mientras que las mínimas fluctúan entre 12 y 23 ${ }^{\circ} \mathrm{C}$ (Adame-Castro et al. 2020). Durante el experimento las temperaturas fluctuaron entre 19.8 y 29.1 ${ }^{\circ} \mathrm{C}$, mientras que las precipitaciones fueron de 102.8 $\mathrm{mm}$ con máximo de $40.8 \mathrm{~mm}$ en $24 \mathrm{~h}$.

\section{Formulación experimental de los tratamientos}

Se formularon tres tratamientos que incluyeron: esquilmos de sorgo, pollinaza, concentrado comercial, con 33\% de sorgo, 33\% de maíz, $16.5 \%$ de pasta de soya, $16.5 \%$ de salvadillo y $1 \%$ de premezcla. Además, en los tratamientos se incluyó heno de pasto mombasa (Panicum maximum cv. mombasa) y ensilado de maíz con diferentes niveles de inclusión (15\% para el T1, 31\% para el T2 y $45 \%$ para el T3) como se muestra en la Tabla 1.

Tabla 1. Inclusión de los insumos de la ración por tratamiento en función al contenido de MS (\%).

\begin{tabular}{lccc}
\hline Insumo & T1 & T2 & T3 \\
\hline Esquilmos de sorgo & 30 & 14 & 0 \\
Pollinaza & 15 & 15 & 15 \\
Concentrado comercial & 18 & 18 & 18 \\
Heno de mombasa & 22 & 22 & 22 \\
Ensilado de maíz & 15 & 31 & 45 \\
\hline T1 $=15 \%$ de ensilado maíz, T2 $=31 \%$ de en- \\
silado maíz, T2 = 45\% de ensilado maíz.
\end{tabular}

Para la formulación de los tratamientos se consideró un consumo de materia seca del $3.2 \%$ del peso vivió de cada animal, además de la etapa de lactación, así también la condición corporal de las vacas siguiendo el procedimiento descrito por PozoLeyva et al. (2021) y NRC, (2001). Antes de iniciar el experimento las nueve vacas se encontraban estabuladas, junto al resto del rebaño, con una ración convencional, la cual se tomó como modelo para la formulación del tratamiento dos (T2) y en función de este se formularon los tratamientos T1 y T3, tomando en cuenta un valor por debajo de lo que dan los productores de manera convencional y aumentando la cantidad de ensilado con el objetivo de determinar la respuesta productiva y económica de la suplementación con ensilaje de maíz siguiendo la metodología de Albarrán-Portillo et al. (2012).

\section{Composición química de los ingredientes de la ración}

A cada ingrediente se le determinó su composición química. Una semana previa al inicio del experimento se tomaron muestras para determinar el contenido de materia seca (MS) mediante secado en estufa de aire forzado a $65{ }^{\circ} \mathrm{C}$ durante $48 \mathrm{~h}$. La materia orgánica $(\mathrm{MO})$ y la ceniza se determinó por incineración en mufla a $550{ }^{\circ} \mathrm{C}$ durante $3 \mathrm{~h}$. Para determinar la proteína cruda ( $P C)$ se empleó la técnica de combustión seca por medio de un Analizador Elemental CHNS/O (Perkin Elmer 2400 Serie II), la fibra detergente neutro (FDN) y la fibra ácido detergente (FDA) por el método de micro bolsas (ANKOM Technology 2005). La digestibilidad in vitro de la materia seca (DIVMS) se determinó mediante incubación en líquido ruminal (Gómez-Miranda et al. 2020).

\section{Variables de respuesta animal}

Se llevó a cabo un ordeño de forma mecanizada a las 8:00 $\mathrm{h}$, donde las vacas fueron suplementadas con $2.3 \mathrm{~kg}$ de pollinaza y $2.6 \mathrm{~kg}$ de concentrado comercial, ambos en materia fresca. Lo que corresponde al 15 y $18 \%$ de inclusión del total de la ración, respectivamente; en fusión al consumo de MS previamente estimado. Posterior al ordeño, cada vaca y su cría se alojaron juntos por aproximadamente $7 \mathrm{~h}$ diarias, donde se suministró de forma individual el resto de la ración, de acuerdo al tratamiento 
correspondiente por animal, periodo experimental y tratamiento. Todos los animales contaron con libre acceso al agua.

La producción de leche se midió después del ordeño durante los cuatro últimos días de cada periodo experimental. Se empleó una balanza digital con capacidad de $30 \mathrm{~kg}$. La composición química de la leche (PC, grasa, densidad y SNG) se determinó empleando un analizador de leche portátil (lacticheck $^{\mathrm{TM}}$, Modelo LC-01), en muestras individuales que se tomaron después del ordeño.

Para conocer el peso vivo (PV) y la condición corporal (CC) de cada animal, se procedió al pesaje de los animales después del ordeño. EI PV y la CC se midieron el día antes de iniciar el experimento y el último día de cada periodo experimental. Para el PV de los animales se empleó una báscula con capacidad de $1000 \mathrm{~kg}$ y la CC se midió mediante la escala de 1 a 5 , donde 1 es muy flaca y 5 es muy gorda (Ferguson et al. 1994).

\section{Análisis costo beneficio de la implementación de los tratamientos}

Para obtener los costos de alimentación por tratamiento se empleó el método de presupuestos parciales, considerando solo los costos de alimentación y el retorno por producción de leche. Como el sistema productivo es familiar no se consideraron costos de mano de obra ni gastos operativos, de acuerdo con lo reportado en trabajos previos (Albarrán-Portillo et al. 2012, López-González et al. 2017). Se consideró el tipo de cambio del dólar estadounidense (US\$) de $\$ 19.20$ pesos mexicanos, según la banca mexicana al momento del experimento.

\section{Diseño experimental}

El experimento tuvo una duración de 36 días, divididos en tres periodos experimentales. La adaptación a cada periodo fue de ocho días y cuatro días para la evaluación de la respuesta animal. Considerando que cuando los cambios en la alimentación no son drásticos es viable el empleo de periodos experimentales cortos (Miguel et al. 2014), además que se reducen costos y el tiempo de manipulación de los animales, lo que es conveniente en investigaciones con productores participantes. Para las variables de respuesta animal se empleó un cuadrado latino $3 \times 3$ triplicado, el segundo cuadro fue en espejo con respecto al primero y el tercer cuadro se volvió a aleatorizar. La asignación de las vacas dentro de los cuadros a la secuencia de tratamiento fue de forma aleatoria y de la misma manera se asignó la secuencia de tratamientos por grupo de animales considerando la producción de leche, el peso vivo y los días en lactancia. La comparación de datos se realizó mediante ANOVA utilizando el software Minitab ${ }^{\circledR}$ V-14. El modelo para el análisis estadístico fue de acuerdo a lo descrito por López-González et al. (2020):

$$
Y_{i j k l}=\mu+C_{i}+V_{j(i)}+P_{k}+t_{l}+e_{i j k l}
$$

Dónde: $\mu=$ Media general; $\mathrm{C}=$ Efecto del cuadro, $(\mathrm{i}$ $=1,2,3) ; \mathrm{V}=$ Efecto vacas dentro del cuadro $(\mathrm{j}=1$, 2, 3...9); $\mathrm{P}=$ Efecto debido al periodo experimental, $(\mathrm{k}=1,2,3) ; \mathrm{t}=$ Efecto de tratamiento $(1,2,3) ; \mathrm{y} \mathrm{e}=$ Error experimental.

\section{RESULTADOS}

\section{Componentes de la ración}

Los resultados de la composición química de los ingredientes de la ración se muestran en la Tabla 2 , el contenido de MS va del 29 al $93 \%$, la MO del 65 al $91 \%$ y la ceniza del 6 al 27\%. Mientras que el contenido de PC de los ingredientes utilizados fluctúo entre 3 y 19\%, los cuales se encuentra relacionados con la cantidad de fibras que contienen. El bajo contenido de proteína cruda de los esquilmos de sorgo (3.44\%), se relacionan con los altos contenidos de FDN y FDA (61.7 y 39.8\%, respectivamente), con DIVMS alta (73.7\%). En cuanto a los demás ingredientes se observó un buen contenido de PC, así como elevado contenido de fibras, con buena digestibilidad. De forma general el contenido de MS y $\mathrm{PC}$ de las raciones en su conjunto se comportó de la siguiente forma: T1 $683 \mathrm{~g} \mathrm{~kg}^{-1}$ de MS y $127 \mathrm{~g} \mathrm{~kg}^{-1}$ de PC, T2 $493 \mathrm{~g} \mathrm{~kg}^{-1}$ de MS y $136 \mathrm{~g} \mathrm{~kg}^{-1}$ de PC y 
Tabla 2. Composición química de los ingredientes de la ración (\%).

\begin{tabular}{lccccccc}
\hline Ingredientes & MS & MO & Ceniza & PC & FDN & FDA & DIVMS \\
\hline Esquilmos de sorgo & 91.19 & 64.68 & 26.51 & 3.44 & 61.70 & 39.80 & 73.10 \\
Pollinaza & 84.21 & 75.57 & 18.98 & 18.79 & 59.70 & 45.50 & 70.70 \\
Concentrado comercial & 90.30 & 90.55 & 7.56 & 15.63 & 32.00 & 12.10 & 89.20 \\
Heno de pasto mombasa & 92.68 & 82.30 & 10.38 & 9.88 & 67.30 & 40.20 & 71.80 \\
Ensilado de maíz & 28.90 & 71.80 & 5.54 & 9.13 & 69.80 & 46.70 & 73.80 \\
Media & 77.46 & 76.98 & 13.79 & 11.37 & 58.10 & 36.86 & 75.72 \\
\hline
\end{tabular}

$\mathrm{MS}=$ materia seca, $\mathrm{MO}=$ materia orgánica, $\mathrm{PC}$ = proteína cruda, $\mathrm{FDN}$ = fibra detergente neutro,

FDA = fibra detergente ácido, DIVMS = digestibilidad in vitro de la materia seca.

T3 $423 \mathrm{~g} \mathrm{~kg}^{-1}$ de MS y $150 \mathrm{~g} \mathrm{~kg}^{-1}$ de PC.

\section{Variables de respuesta animal}

Como se observa en la Tabla 3 solo mostraron diferencias $(P<0.05)$ la condición corporal, siendo favorable para el T3. La producción de leche promedio por vaca al día fue de $4.4 \mathrm{~kg}$, con contenido de grasa del $3.31 \%$, densidad de $30.23 \%$, mientras que la PC fue del $3.5 \%$ y los SNG del $8.8 \%$. El PV fue de $425 \mathrm{~kg}$ con una condición corporal de 2.3 puntos. No se observaron efectos de los periodos experimentales ni de la interacción periodo por tratamiento para ninguna de las variables evaluadas.

\section{Análisis costo beneficio de la implementación de los tratamientos}

Con relación al análisis económico presentado en la Tabla 4, se observa que el T2 tuvo un incremento de $\$ 5.76$ en los costos de alimentación con respecto al T1, mientras que el T3 tuvo un incremento de \$11.16. La incorporación del ensilado de maíz en 15, 31 y $45 \%$, mostró que aumenta el 0.36, 0.72 y $1.07 \%$ los costos totales de alimentación, sin efecto sobre la producción de leche. El costo de producción de la leche fue de $0.32,0.35$ y $0.38 \$ \mathrm{~kg}^{-1}$ para el T1, T2 y T3, respectivamente con margen de ganancia promedio de $0.1 \$ \mathrm{~kg}^{-1}$.

\section{DISCUSIÓN}

\section{Componentes de la ración}

Para la elaboración de los tratamientos se consideró el empleo de productos locales de fácil adquisición en la zona de estudio. Se emplearon subproductos de la producían agropecuaria como el esquilmo de sorgo y la pollinaza, bajo el principio planteado por
Bakyusa-Katongole et al. (2016), quienes describen que el empleo de subproductos de cosecha en la alimentación animal es una estrategia viable para reducir costos de producción, ya que gran parte de estos subproductos, son quemados o quedan en los sitios de cultivo sin ningún empleo productivo. Por lo que pequeños y medianos productores recuperan estos subproductos para incorporarlos en la producción animal. Además, de que uno de los cultivos que genera gran cantidad de residuos es el del sorgo, el cual puede ser incorporado a la alimentación animal de pequeños y medianos productores. Mientras que Absalón-Medina et al. (2012) y Citalan-Cifuentes et al. (2016) reportaron que es recomendable el empleo de los subproductos de la producción de aves, los cuales generalmente contienen purines, mezcla de estiércol, orina, cama, restos de alimentos y plumas; que tienen un alto nivel proteico y costo de adquisición menor al del concentrado comercial. Por otro lado, González-Alcántara et al. (2020) concuerdan con la viabilidad de conservar alimentos para suplementar a los animales durante la temporada seca, como puede ser el heno y el ensilado. Además, reportan que el principal producto a ensilar en los sistemas de producción de leche en pequeña escala del centro de México es el maíz, el cual ha sido implementado por el $30 \%$ de los productores como trasferencia tecnológica ante la limitada calidad de los pastos durante la temporada seca. Sin embargo, en la región sureste de México la información referente al uso del ensilado de maíz en los sistemas de producción de leche es limitada, tal como lo señalan Robles-Jiménez et al. (2021) quienes estudiaron el rendimiento y la calidad del forraje del ensilaje de maíz producido en México y su potencial en la producción de leche.

La composición química de los ingredientes 
Tabla 3. Producción de leche, composición química y variables de respuesta animal por tratamiento.

\begin{tabular}{lccccccccc}
\hline Variables & T1 & T2 & T3 & Media & EEM & P1 & P2 & P3 & EEM \\
\hline Producción de leche $\left(\mathrm{kg} \mathrm{vaca}^{-1}\right.$ día $\left.^{-1}\right)$ & 4.32 & 4.40 & 4.57 & 4.43 & $0.55^{N S}$ & 4.89 & 4.16 & 4.21 & $0.55^{N S}$ \\
Peso vivo $\left(\mathrm{kg} \mathrm{vaca}^{-1}\right.$ ) & 412.30 & 428.30 & 433.10 & 424.60 & $25.59^{N S}$ & 423 & 427 & 421 & $25.59^{N S}$ \\
Condición corporal & 2.00 & 2.30 & 2.50 & 2.30 & $0.18^{\star *}$ & 2.16 & 2.47 & 2.16 & $0.18^{\star *}$ \\
PC (\%) & 3.39 & 3.53 & 3.45 & 3.46 & $0.09^{N S}$ & 3.44 & 3.40 & 3.49 & $0.09^{N S}$ \\
Grasa (\%) & 3.31 & 3.25 & 3.37 & 3.31 & $0.40^{N S}$ & 3.05 & 3.22 & 3.65 & $0.40^{N S}$ \\
Densidad (\%) & 30.01 & 30.64 & 30.04 & 30.23 & $0.70^{N S}$ & 31.85 & 29.30 & 29.48 & $0.70^{N S}$ \\
SNG (\%) & 9.47 & 8.51 & 8.49 & 8.82 & $0.19^{N S}$ & 9.4 & 8.47 & 8.49 & $0.19^{N S}$
\end{tabular}

$\mathrm{T}=$ Tratamiento 1, 2, 3, EEM = error estándar de la media, $\mathrm{PC}=$ proteína cruda $\mathrm{SNG}=$ Solidos no grasos, $\mathrm{P}=$ periodo de experimentación 1, 2, 3. NS $P>0.05,{ }^{*} P<0.05,{ }^{* *} P<0.01,{ }^{* * *} P<0.001$.

\begin{tabular}{|c|c|c|c|}
\hline Variable & $\mathrm{T} 1$ & T2 & T3 \\
\hline \multicolumn{4}{|l|}{ Costos de alimentación (US\$/kg/MS) } \\
\hline Esquilmos de sorgo & 0.01 & 0.01 & 0.01 \\
\hline Pollinaza & 0.06 & 0.06 & 0.06 \\
\hline Concentrado comercial & 0.43 & 0.43 & 0.43 \\
\hline Heno de pasto mombasa & 0.01 & 0.01 & 0.01 \\
\hline Ensilado de maíz & 0.09 & 0.09 & 0.09 \\
\hline \multicolumn{4}{|c|}{ Costos de alimentación por vaca al día (US \$/ kg/ MS/tratamiento) } \\
\hline Esquilmos de sorgo & 0.05 & 0.02 & 0 \\
\hline Pollinaza & 0.12 & 0.12 & 0.12 \\
\hline Concentrado comercial & 1.02 & 1.02 & 1.02 \\
\hline Heno de pasto mombasa & 0.03 & 0.03 & 0.03 \\
\hline Ensilado de maíz & 0.18 & 0.36 & 0.54 \\
\hline Costo de la ración (\$/día) & 1.39 & 1.55 & 1.70 \\
\hline Costo total por tratamiento & 50.04 & 55.80 & 61.20 \\
\hline \multicolumn{4}{|l|}{ Retornos (US \$) } \\
\hline Producción de leche (kg/vaca en 36 días) & 155.52 & 158.4 & 164.52 \\
\hline Precio de venta (kg/días) & 0.42 & 0.42 & 0.42 \\
\hline Retorno por ventas de leche & 65.32 & 66.53 & 69.09 \\
\hline El costo de producción por $\mathrm{kg}$ de leche $(\$ / \mathrm{kg})$ & 0.32 & 0.35 & 0.37 \\
\hline Margen sobre el costo de alimentación & 14.28 & 10.73 & 7.89 \\
\hline Rentabilidad por kg de leche & 0.09 & 0.07 & 0.05 \\
\hline
\end{tabular}

de la ración coincide con lo reportado en investigaciones previas que reportan contenidos de $8.3 \%$ de PC, $23.5 \%$ de MS, 33.20 de FDN y $68.6 \%$ de FDA para el ensilado de maíz; en el caso del concentrado comercial $17.03 \%$ de PC, $92.9 \%$ de MS, y $9.7 \%$ de FDA; y la Pollinaza con $20.4 \%$ de PC, $82.0 \%$ de MS, y $39.1 \%$ de FDA (Absalón-Medina et al. 2012). Mientras que para el sorgo se reportan valores de $33 \%$ de PC, $74.3 \%$ de MS, 65.8 de FDN y $42.6 \%$ de FDA (Bakyusa-Katongole et al. 2016). Por lo que con excepción del concentrado comercial el resto de los ingredientes de la ración se pueden considerar alimentos de mediana calidad, ya que para considerar un alimento de alta calidad se requiere que tenga contenidos de PC superiores al 15\% y la FDN menor al $50 \%$, ya que mayores concentraciones de FDN dan como resultado una menor digestibilidad de los alimentos (Partida-Hernández et al. 2019). Considerando lo anterior se podría inferir que las raciones con mayor suplementación de ensilado como en el caso del T3 tendrían mayor digestibilidad y mayor aporte de nutrientes digestibles, en relación a las de menor concentración de ensilado y mayor contenido de esquilmo de sorgo T1.

\section{Variables de respuesta animal}

Las variables de respuesta animal se mantuvieron de forma similar al preexperimental y a pesar de que los rendimientos de leche fueron moderados estos no disminuyeron en el tiempo. Entre los fac- 
tores que pueden afectar la producción y composición de la leche se encuentra la genética animal y la época del año, ya que en la temporada seca que comprende de diciembre a mayo disminuye la disponibilidad de pastos y aumenta el estrés por calor, siendo más susceptibles en los animales de mayor potencial genético (González-Alcántara et al. 2020), lo que pudiera explicar los resultados encontrados en este experimento. De hecho, en las investigaciones previas (Ramírez-Rivera et al. 2019, Pozo-Leyva et al. 2019, Pozo-Leyva et al. 2021), se ha observado heterogeneidad en la composición de las raciones mixtas que incluyen diferentes productos derivados del maíz, así como los indicadores productivos. Lo anterior, puede estar determinado por las características de los animales, las particularidades de las zonas agroecológicas donde se encuentre los sistemas productivos, así como de los hábitos y costumbres de los productores participantes.

En un estudio efectuado por AlbuquerqueSaraiva et al. (2020) reporta una producción de leche de $16 \mathrm{~kg} \mathrm{día}^{-1}$, con contenido de grasa entre el 3.8 y $4.4 \%$, PC entre 3.7 y $3.9 \%$, en vacas Holstein de $670 \mathrm{~kg}$ de peso vivo. Mientras que GonzálezAlcántara et al. (2020) evaluaron el suministro de ensilado de $X$. Triticosecale en vacas Holstein durante la estación seca con una producción de leche de $12 \mathrm{~kg} \mathrm{vaca}^{-1}$ días $^{-1}$, contenido de grasa de $3.4 \%$, PC del 3.3\% y condición corporal de 2.4 puntos. En ambas investigaciones a pesar de ser efectuados en diferentes condiciones ecológicas y productivas no se documentó diferencias significativas entre los tratamientos empleados lo que coincide con los resultados encontrados en el presente experimento. Por otra parte, estos mismos autores al igual que lo encontrado en esta investigación reportaron que las variables de respuesta animal se mantuvieron en el tiempo con una producción y composición química de la leche similar a la producción preexperimental. Los resultados de las variables de respuesta encontrados son menores a los reportados por Albuquerque-Saraiva et al. (2020) y González-Alcántara et al. (2020), lo que puede estar influenciado por la genética animal, el nivel de tecnificación y las características agroecológicas del sitio de estudio.

En investigaciones que emplean animales provenientes de múltiples cruzas con las razas locales en zonas tropicales tanto los indicadores productivos como la composición de la leche están más acordes con los aquí reportados. Tal es el caso de la investigación efectuada por Camero et al. (2001) quienes evaluaron el comportamiento de vacas mestizas con razas locales del trópico de Costa Rica suplementadas con E. poeppigiana o G. sepium, reportando una producción de leche de $7 \mathrm{~kg}$ leche vaca ${ }^{-1}$ día $^{-1}$. Al igual que Salvador-Loreto et al. (2016) con vacas pardo suizo, suplementados con subproductos de cosecha, reportan una producción de leche de 7 $\mathrm{kg} \mathrm{vaca}^{-1}$ día $^{-1}$ con contenido de grasa y de PC del 3.1 y $3.0 \%$, respetivamente. Mientras que BakyusaKatongole et al. (2016) al evaluar la inclusión de heno de $P$. maximum en la alimentación de vacas frisonas de cruces con razas locales, reportan una producción de leche diaria de entre 6 y $7 \mathrm{~kg} \mathrm{vaca}^{-1}$. Aunque durante el experimento las temperaturas fluctuaron entre 20 y $29^{\circ} \mathrm{C}$, lo que pudo provocar estrés por calor y a pesar del correcto suministro de MS y PC no se reflejó en los indicadores productivos.

La producción de leche, el peso vivo y la condición corporal se mantuvieron constantes, incluso con una pequeña tendencia al alza del T3 con respecto a $\mathrm{T} 1$ lo que difiere de otras investigaciones efectuadas en condiciones tropicales, que reportan que es común observar una disminución del peso y la condición corporal durante la temporada seca (Absalón-Medina et al. 2012, Ramírez-Rivera et al. 2019). Debido a que las vacas en producción de leche movilizan hasta un $25 \%$ de su peso vivo para la producción de leche, lo que influye en los indicadores productivos y la salud. Lo anterior, indica que las raciones empleadas constituyen una alternativa de alimentación para contrarrestar los efectos de la época del año, con respecto a la producción animal en condiciones agroecológicas y productivas semejantes a las del sitio de estudio. El mantenimiento de la producción de leche y el ligero incremento de la condición corporal pudo estar influenciado por la ineficiencia de los bovinos en convertir la PC consumida en leche, la cual es del $15 \%$ aproximada- 
mente, además de la movilización de nutrientes para el mantenimiento de la gestación (Pozo-Leyva et al. 2021). Lo que pudo intervenir en una mayor percepción del aumento de la condición corporal sin verse reflejado en una ganancia de peso.

\section{Análisis costo beneficio de la implementación de los tratamientos}

En promedio el concentrado comercial representó el $65.8 \%$ de los costos diarios de alimentación, lo que coincide con los resultados reportados por Albarrán-Portillo et al. (2012) y López-González et al. (2017). En el presente estudio, un factor que limito los márgenes de ganancia fue la producción de leche, por lo que las estrategias de gestión productivas deberán ir encaminadas a incrementar la producción de leche diaria además de reducir los costos por el ingreso de productos externos como el concentrado comercial (Pozo-Leyva et al. 2019).

\section{CONCLUSIONES}

La incorporación del ensilado de maíz en un 15, 31 o $45 \%$ vaca $^{-1}$ día $^{-1}$ en las raciones mixtas de vacas doble propósito con producción de $4.4 \mathrm{~kg}$ leche ${ }^{-1}$ día $^{-1}$ no incrementó su producción, pero si tienen efecto en los costó total de alimentación lo que reduce el margen de ganancia para T2 y T3. Bajo las condiciones de estudió se recomienda el empleó de una ración con $15 \%$ de ensilado de maíz, 30\% de esquilmos de sorgo, $18 \%$ de concentrado comercial, $15 \%$ de pollinaza y $22 \%$ de heno de mombasa.

\section{AGRADECIMIENTOS}

Este artículo es resultado del proyecto: 316492, apoyado por el Consejo Nacional de Ciencia y Tecnología (CONACYT) en el año 2021. Asimismo, al CONACYT por la Beca Posdoctoral otorgada al Dr. Dixan Pozo-Leyva (Clave: BPPA-20200622155429245-364115). Del mismo modo agradecemos a los dos productores que participaron en este estudio. También a la Ing. Carolina Yam-Chalé por su asistencia técnica en los análisis de laboratorio y a los estudiantes de ingeniería en agronomía Dina Laura Méndez-Cervada y Jesús Humberto Colli-Alvarado.

\section{LITERATURA CITADA}

Absalón-Medina VA, Blake-Robert W, Gene-Fox D, Juárez-Lagunes FI, Nicholson ChF, Canudas-Lara EG, RuedaMaldonado BL (2012) Limitations and potentials of dual-purpose cow herds in Central Coastal Veracruz, Mexico. Tropical Animal Health and Production 44: 1131-1142.

Adame-Castro DE, Aryal DR, Villanueva-López G, López-Martínez LO, Chay-Canul AJ, Casanova-Lugo F (2020) Diurnal and seasonal variations on soil $\mathrm{CO}_{2}$ fluxes in tropical silvopastoral systems. Soil Use and Management 36: 671-681.

Albarrán-Portillo B, García A, Espinoza A, Espinosa E, Arriaga CM (2012) Maize silage in the dry season for grazing dairy cows in small-scale production systems in Mexicos Highlands. Indian Journal of Animal Research 46: 317-324.

Albarrán-Portillo B, Rebollar-Rebollar S, García-Martínez A, Rojo-Rubio R, Avilés-Nova F, Arriaga-Jordán CM (2015) Socioeconomic and productive characterization of dual-purpose farms oriented to milk production in a subtropical region of Mexico. Tropical Animal Health and Production 47: 519-523.

Albuquerque-Saraiva T, Figueiredo-Monteiro C, Santos-Feitosa EM, Oliveira-Moraes GS, Joelson-Netto A, BarrosCardoso D, Rodrigues-Magalhães AL, Silva-de-Melo AA (2020) Effect of association of fresh cassava root with corn silage in replacement for cactus cladodes on dairy cow performance. Tropical Animal Health and Production 52: 927-933. 
Ankom Technology (2005) Procedures (for NDF and ADF). In vitro true digestibility using the DAISY II incubator. http://www.ankom.com. Fecha de consulta: 20 de octubre de 2020.

Arjona-Alcocer VA, Aguilar-Pérez CF, Ku-Vera JC, Ramírez-Avilés L, Solorio-Sánchez FJ (2020) Influence of energy supplementation on dietary nitrogen utilization and milk production in cows fed foliage of Leucaena leucocephala. Tropical Animal Health and Production 52: 2319-2325.

Bakyusa-Katongole C, Mary-Kabirizi J, Ntege-Nanyeenya W, Kigongo J, Nviiri G (2016) Milk yield response of cows supplemented with sorghum stover and Tithonia diversifolia leaf hay diets during the dry season in northern Uganda. Tropical Animal Health and Production 48: 1463-1469.

Camero A, Ibrahim M, Kass M (2001) Improving rumen fermentation and milk production with legume-tree fodder in the tropics. Agroforestry Systems 51: 157-166.

Citalan-Cifuentes LH, Ramos-Juárez JA, Salinas-Hernández R, Bucio-Galindo A, Herrera-Haro JG, OrantesZebadua MA (2016) Análisis sensorial de leche de vacas suplementadas con un alimento fermentado a base de pollinaza. Ecosistemas y Recursos Agropecuarios 3: 181-191.

Espinoza-Ortega A, Espinosa-Ayala E, Bastida-López J, Castañeda-Martínez T, Arriaga-Jordán CM (2007) Smallscale dairy farming in the highlands of Central Mexico: technical, economic and social aspects and their impact on poverty. Experimental Agriculture 43: 241-256.

Ferguson JD, Galligan DT, Thomsen N (1994) Principal descriptors of body condition score in Holstein cows. Journal of Dairy Science 77: 2695-2703.

Gómez-Miranda A, Vega-García JI, Narvaez-Uribe O, Morales-Almaraz E, López-González F, Arriaga-Jordán CM (2020) Evaluación de un concentrado de pasta de canola y mazorca de maíz para vacas lecheras en pastoreo de pastizales nativos en los Valles Altos de México. Tropical and Subtropical Agroecosystems 23: \#58.

González-Alcántara FJ, Estrada-Flores JG, Morales-Almaraz E, López-González F, Gómez-Miranda A, VegaGarcía JI, Arriaga-Jordán CM (2020) Whole-crop triticale silage for dairy cows grazing perennial ryegrass (Lolium perenne) or tall fescue (Lolium arundinaceum) pastures in small-scale dairy systems during the dry season in the highlands of Mexico. Tropical Animal Health and Production 52: 1903-1910.

López-González F, Cantú-Patiño MG, Gama-Garduño O, Prospero-Bernal F, Colín-Navarro V, Arriaga-Jordán CM (2020) Praderas de festuca alta y ryegrass en pastoreo de vacas lecheras en sistemas de producción de leche en pequeña escala en los Valles Altos del Centro de México.Tropical and Subtropical Agroecosystems 23: \#39.

López-González F, Rosas-Dávila M, Celis-Alvarez MD, Morales-Almaraz E, Domínguez-Vara IA, ArriagaJordán CM (2017) Milk production under grazing of different pasture grasses in small-scale dairy systems in the highlands of central Mexico. Livestock Science Journal 8: 92-97.

Miguel MF, Ribeiro-Filho HMN, De Andrade EA, Moraes Genro MT, Delagarde R (2014) Pasture intake and milk production of dairy cows grazing annual ryegrass with or without corn silage supplementation, Animal Production Science 54: 1810-1816.

NRC (2001) Nutrient requirements of dairy cattle. 7th Revised Edition. National Research Council. National Academy Press, Washington, DC, USA. 405p.

Partida-Hernández M, Loya-Olguin JL, Gómez-Gurrola A, Ramírez-Ramírez JC, Hernández-Ballesteros JA, Teresita Amezcua-Jaeger T, Escalera-Valente F, Sanginés-García L (2019) Reemplazo de grano de sorgo 
con fruto de Guazuma ulmifolia en dietas de corderos con diferente forraje. Ecosistemas y Recursos Agropecuarios 6: 253-262.

Pozo-Leyva D, López-González F, Casanova-Lugo F, Carrillo-Hernández S, Velarde-Guillen J, Arriaga-Jordán CM (2021) Nitrogen use efficiency and soil chemical composition in small-scale dairy systems. Tropical Animal Health and Production 53: 538. DOI: doi.org/10.1007/s11250-021-02988-6.

Pozo-Leyva D, López-González F, Olea-Pérez R, Balderas-Hernández P, Arriaga-Jordán CM (2019) Nitrogen utilization efficiency in small-scale dairy systems in the highlands of central Mexico. Tropical Animal Health and Production 51: 1215-1223.

Ramírez-Rivera EJ, Rodríguez-Miranda J, Huerta-Mora IR, Cárdenas-Cágal A, Juárez-Barrientos JM (2019) Tropical milk production systems and milk quality: a review. Tropical Animal Health and Production 51: 1295-1305.

Robles-Jiménez LE, Rosas-Dávila M, Osorio-Avalos J, Chay-Canul AJ, Palacios-Riocerezo C, Castelán-Ortega OA, González-Ronquillo M (2021) Evaluation of Mexican native and hybrid maize (Zea mays silages for sustainable milk production. Tropical and Subtropical Agroecosystems 24: 1-10.

Rojo-Rubio R, Vázquez-Armijo JF, Pérez-Hernández P, Mendoza-Martínez GD, Salem AZM, Albarrán-Portillo B, González-Reyna A, Hernández-Martínez J, Rebollar-Rebollar S, Cardoso-Jiménez D, Dorantes-Coronado EJ, Gutierrez-Cedillo JG (2009) Dual purpose cattle production in Mexico. Tropical Animal Health and Production 41: 715-721.

Salvador-Loreto I, Arriaga-Jordán CM, Estrada-Flores JG, Vicente-Mainar F, García-Martínez A, Albarrán-Portillo B (2016) Molasses supplementation for dual-purpose cows during the dry season in subtropical Mexico. Tropical Animal Health and Production 48: 643-648.

SIAP (2014) Estadísticas del sector ganadero. Secretaría de Agricultura Ganadería Desarrollo Rural Pesca y Alimentación. Sistema de Información y Estadística Agropecuaria y Pesquera. México. www.sagarpa.gob.mx/. Fecha de consulta: 10 de noviembre de 2021 